\title{
Clinical Trials, Emerging Neuropsychopharmacology, and Structural Neuroanatomy for the Practicing Psychiatrist and Neurologist
}

\author{
By Eric Hollander, MD
}

This month's CNS Spectrums highlights the emerging role of the cannabinoid system for breakthrough treatments in anxiety and fear, describes targeted treatments of childhood depression and the affective instability domain in borderline personality disorder, and describes the neural circuitry of fear conditioning and extinction.

Kevin A. Corcoran, PhD, and Gregory J. Quirk, $\mathrm{PhD}$, review how persistent fear and anxiety following exposure therapy could reflect a deficit in the recall of extinction learning. A neural circuit for extinction learning and recall that includes the amygdala, ventromedial prefrontal cortex, and hippocampus has been identified. Of interest, the ventromedial prefrontal cortex is a site of neural plasticity that allows for the inhibition of fear during extinction recall, and receives information from other brain regions, such as contextual information from the hippocampus, to determine the circumstances under which extinction or fear will be recalled. Understanding the neural circuitry underlying extinction recall may lead to more effective therapies for disorders of fear and anxiety.

Wendy Weinstein, MD, and Katrina L. Jamison, PharmD, utilized a chart review to study the use of lamotrigine to treat symptoms of affective instability in patients with borderline personality disorder. The charts of 13 patients were reviewed and included in the analysis. All patients were female and had reported continuing symptoms of affective instability despite treatment with two to seven psychotropic drugs. Lamotrigine seemed to be a safe and effective option for the treatment of patients with symptoms of affective instability associated with borderline personality disorder, but controlled trials are needed to confirm these observations.

Jasmeer P. Chhatwal, PhD, and Kerry J. Ressler, MD, PhD, review progress in the understanding of the mammalian cannabinoid system, from the cloning of the endogenous cannabinoid receptor to the discovery of new pharmacologic compounds acting on this receptor. This has resulted in a greater understanding of the neural circuitry that mediates anxiety and fear responses, and the acquisition, expression, and extinction of conditioned fear as a model of human anxiety. This may provide important emerging targets for new treatments of anxiety disorders.

Graham J. Emslie, MD, Paul P. Yeung, MD, $M P H$, and Nadia R. Kunz, PharmD, describe a trial of the long-term effectiveness and safety of venlafaxine extended-release in children and adolescents with major depressive. Most of the improvement with venlafaxine extended-release was found to occur during the first 6 weeks of treatment. It is worth noting that clinicians need to monitor for signs of suicidal ideation and hostility in pediatric patients treated with all antidepressants, especially on initiating treatment, and with dosage adjustments.

Dr. Hollander is the editor of this journal, Esther and Joseph Klingenstein Professor and Chairman of Psychiatry at the Mount Sinai School of Medicine, and director of the Seaver and New York Autism Center of Excellence in New York City. 
In addition, Michael Trimble, MD, FRCP, FRPsych, introduces the new "Brain Regions of Interest" series, in which connectivity and functional relationships between several neuroanatomical structures are highlighted. At CNS Spectrums, we hope that this new series will provide practicing psychiatrists and neurologists with a better intuitive feel for these brain structures and systems, as if they had observed actual brain dissections of these neuroanatomic regions of interest. CNS

\section{ERRATUM}

In CNS Spectr. 2007;13(2):113-123, "Alzheimer's Disease: Progress in the Development of Anti-amyloid Disease-Modifying Therapies," Christensen DD, the generic name of Alzhemed is incorrectly listed as "transiposate". The correct spelling of the generic name is tramiprosate. We apologize for the error.

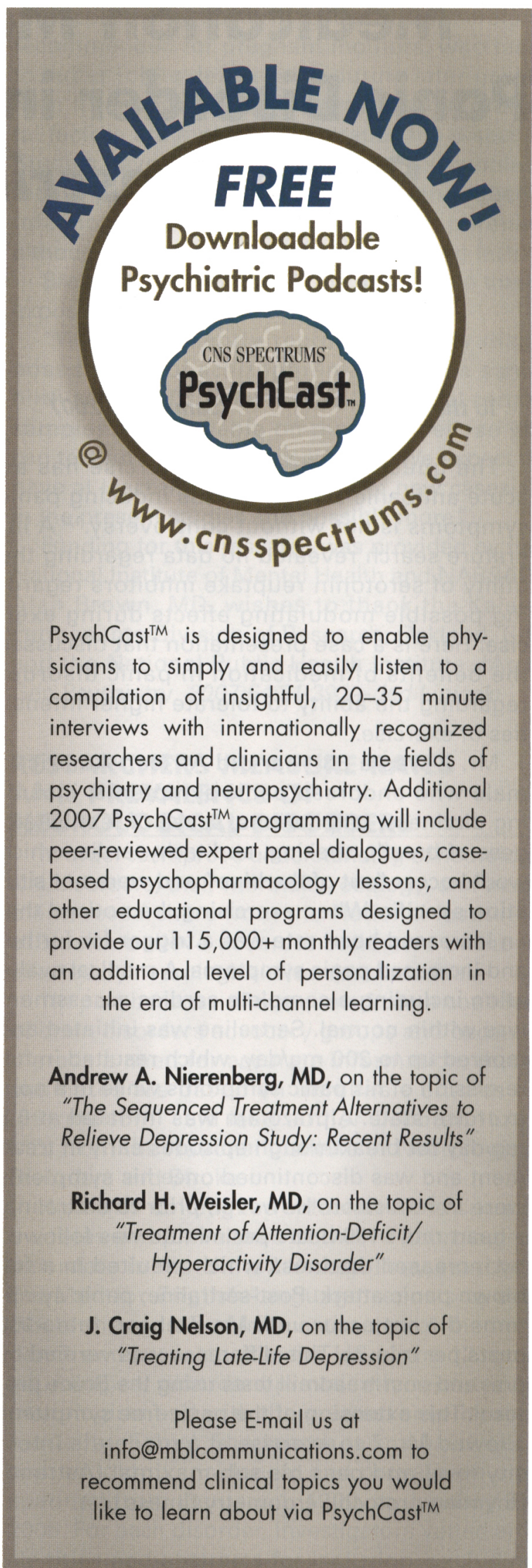

